Nr. $195 / 95$

On the Martingale Property for Generalized

Stochastic Processes

F. E. Benth and J. Potthoff 


\title{
On the Martingale Property for Generalized Stochastic Processes
}

\author{
Fred E. Benth ${ }^{1}$ AND Jürgen PotThofF ${ }^{2}$
}

\section{Introduction and preliminaries}

In the recent years, several groups have studied stochastic equations (e.g., SDE's, SPDE's, stochastic Volterra equations) outside the framework of the Itô calculus. Often, this led to solutions in spaces of generalized random processes or fields. It is therefore of interest to study the probabilistic properties of generalized stochastic processes, and the present paper makes some (rather naive) first steps into this direction.

If we think of a generalized process as a mapping from the real line (or an interval) into a space of generalized random variables (with some additional properties), then there is a wide range of choices for the latter: e.g., the space $(\mathcal{S})^{*}$ of Hida distributions (e.g. [HKPS]), the space $(\mathcal{S})^{-1}$ of Kondratiev distributions (e.g. [KLS]), the Sobolev type space $\mathcal{D}^{*}$ which is used within the Malliavin calculus and so on. Often, the generalized processes coming up in applications have a chaos decomposition with kernels which belong to $L^{2}\left(\mathbb{R}^{n}\right)$, and in this paper we shall focus our attention on this situation. It will be convenient to work with a space $\mathcal{G}^{*}$ which is larger than $\mathcal{D}^{*}$. This space has already been considered by several authors, cf. e.g. [PT] and the references quoted there. It turns out, that basic notions from the theory of stochastic processes like conditional expectation, martingales, sub- (super-) martingales etc. have a rather natural generalization to mappings from the real line into $\mathcal{G}^{*}$.

The paper is organized as follows. In Section 2 we shall give a construction of the Itô integral (with respect to Brownian motion) of generalized stochastic processes, and compare it with the Hitsuda--Skorokhod integral (e.g. [HKPS]). In Section 3 we define generalized martingales and derive a number of properties. In particular, we prove that the generalized Itô integrals of Section 2 are indeed generalized martingales. Moreover, a representation of generalized martingales (in analogy with the Clark-Haussmann formula) will be shown, and we prove that the Wick product of two generalized martingales is again one. Finally, in Section 4 we define the notion of a generalized semimartingale and give a class of examples. In the remainder of this Introduction we provide the necessary background.

\footnotetext{
${ }^{1}$ Matematisk Institutt, Universitet i Oslo, Norway, and Lehrstuhl für Mathematik V, Universität Mannheim, Germany. Supported by the Norwegian Research Council (NFR) under grant NAVF: $100549 / 410$

${ }^{2}$ Lehrstuhl für Mathematik V, Universität Mannheim, Germany. Partially supported by the Deutsche Forschungsgemeinschaft (DFG).
} 
We shall work within the framework of white noise analysis (e.g., [HKPS]), and choose as basic probability space $\left(\mathcal{S}^{\prime}(\mathbb{R}), \mathcal{F}, \mu\right)$, where $\mathcal{S}^{\prime}(\mathbb{R})$ is the Schwartz space of tempered distributions equipped with the weak topology. $\mathcal{F}$ is the Borel $\sigma$-algebra and $\mu$ is the Gaussian probability measure with characteristic function $\exp \left(-\frac{1}{2}|\cdot|_{2}^{2}\right)$, where $|\cdot|_{2}$ is the $L^{2}(\mathbb{R})$-norm. Introduce on this probability space a Brownian motion $B_{t}$ defined as

$$
B_{t}(\omega):=\left\langle\omega, \mathbf{1}_{[0, t)}\right\rangle, \quad \omega \in \mathcal{S}^{\prime}(\mathbb{R}),
$$

where $\mathbf{1}_{[0, t)}$ is the indicator function of $[0, t)$. The Brownian motion $B_{t}$ is to be understood as the $L^{2}(\mu)$-limit of a sequence of coordinate processes $\left\langle\cdot, \phi_{n}\right\rangle$, where $\phi_{n}$ is a sequence of Schwartz functions converging to $1_{[0, t)}$ in $L^{2}(\mathbb{R})$. Denote the $\sigma$-algebra generated by $B_{s}, 0 \leq s \leq t$, by $\mathcal{F}_{t}$.

Next we quickly recall the construction of the triple of random variables

$$
\mathcal{G} \subset L^{2}(\mu) \subset \mathcal{G}^{*}
$$

which will be at the basis of this paper. (Throughout we shall work with real spaces of random variables. Extensions are made by obvious modifications.) Let $N$ denote the number (or Ornstein-Uhlenbeck) operator, and for $\lambda \geq 0$ denote by $\mathcal{G}_{\lambda}$ the $L^{2}(\mu)$-domain of $\exp (\lambda N)$. With its natural norm $\|\cdot\|_{\lambda}:=$ $\|\exp (\lambda N) \cdot\|_{L^{2}(\mu)}, \mathcal{G}_{\lambda}$ is a Hilbert space. $\mathcal{G}$ denotes the projective limit of the family $\left\{\mathcal{G}_{\lambda}, \lambda \geq 0\right\}, \mathcal{G}^{*}$ denotes its dual. $\mathcal{G}^{*}$ is equal to the inductive limit of the family $\left\{\mathcal{G}_{-\lambda}, \lambda \geq 0\right\}$, where $\mathcal{G}_{-\lambda}$ is the dual of $\mathcal{G}_{\lambda}$. The latter is a Hilbert space with norm denoted by $\|\cdot\|_{-\lambda}$. Upon the usual identification of $L^{2}(\mu)$ with its dual we obtain the triple above. Dual pairing between $\mathcal{G}^{*}$ and $\mathcal{G}$ will be denoted by $\langle(\cdot, \cdot\rangle\rangle$.

It is plain to see that $\mathcal{G}^{*}$ inherits from $\mathcal{G}$ a chaos decomposition: $\Phi \in \mathcal{G}^{*}$ is in one to one correspondence with a sequence $\left(\Phi^{(n)}, n \in N_{0}\right)$, where for $n \in \mathbb{N}_{0}$, $\Phi^{(n)}$ belongs to the symmetric subspace of $L^{2}\left(\mathbb{R}^{n}\right)$, and we may write

$$
\Phi=\sum_{n=0}^{\infty} I_{n}\left(\Phi^{(n)}\right)
$$

where the sum converges in $\mathcal{G}_{\lambda}$ for some large enough $\lambda$. We shall refer to the $\Phi^{(n)}$ also as the kernels of $\Phi$.

By construction we have that $\mathcal{G}$ is invariant under the family operators $\{\exp (\lambda N), \lambda \in \mathbb{R}\}$. The same is true for $\mathcal{G}^{*}$, where the action of $\exp (\lambda N)$ on $\Phi \in \mathcal{G}^{*}$ is defined by duality: it is trivial to verify that the mapping $\varphi \mapsto\langle\langle\Phi, \exp (\lambda N) \varphi\rangle\rangle$ is linear and continuous on $\mathcal{G}$ and we denote the corresponding element in $\mathcal{G}^{*}$ by $\exp (\lambda N) \Phi$. Note that it has kernels $\exp (\lambda n) \Phi^{(n)}$ if the kernels of $\Phi$ are $\Phi^{(n)}$. It is easy to check that for any $\lambda, \nu \in \mathbb{R}$ one has indeed $\exp (\lambda N) \mathcal{G}_{\nu}=\mathcal{G}_{\nu-\lambda}$. Thus we have in particular the relations $\mathcal{G}_{-\lambda}=\exp (\lambda N) L^{2}(\mu), \exp (-\lambda N) \mathcal{G}_{-\lambda}=L^{2}(\mu)$, which we shall use frequently. 
Finally, we recall the definition of the $S$-transform of an element $\Phi$ in $\mathcal{G}^{*}$. It is the following function $S \Phi$ on $\mathcal{S}(\mathbb{R})$ :

$$
S \Phi(\xi):=\left\langle\left\langle\Phi, e^{\langle, \xi\rangle-1 / 2|\xi|_{2}^{2}}\right\rangle\right\rangle, \quad \xi \in \mathcal{S}(\mathbb{R}) .
$$

For $\Phi$ as above one obtains:

$$
S \Phi(\xi)=\sum_{n=0}^{\infty} \int_{\mathbb{R}^{n}} \Phi^{(n)}(u) \xi^{\otimes n}(u) d u .
$$

The Wick product $\Phi \diamond \Psi, \Phi, \Psi \in \mathcal{G}^{*}$, is defined as follows: $S^{-1}(S \Phi \cdot S \Psi)$ and it can be shown [PT] that it defines a continuous mapping from $\mathcal{G}^{*} \times \mathcal{G}^{*}$ into $\mathcal{G}^{*}$.

In the sequel let $I$ denote the positive halfline or a finite interval $[0, T]$, $T<\infty$. By a generalized stochastic process we mean a mapping from $I$ into $\mathcal{G}^{*}$, $t \mapsto \Phi_{t}$, for which there exists a $\lambda \geq 0$, independent of $t$, so that $\Phi_{t} \in \mathcal{G}_{-\lambda}$ for all $t \in I$. Note that this implies that $e^{-\lambda N} \Phi_{t}$ is a stochastic process in $\left(L^{2}\right)$. A generalized stochastic process is said to be jointly measurable if the mapping $(t, \omega) \mapsto e^{-\lambda N} \Phi_{t}(\omega)$ from $I \times \mathcal{S}^{\prime}(\mathbb{R})$ into $\mathbb{R}$ is $\mathcal{B}_{I} \otimes \mathcal{F}$-measurable, where $\mathcal{B}_{I}$ is the Borel $\sigma$-algebra on $I$. All generalized stochastic processes considered in this paper are assumed to be jointly measurable.

We introduce the notion of adaptedness for generalized stochastic processes: Let $X_{t}$ be a stochastic process in $\left(L^{2}\right)$. We know that it has a chaos decomposition $X_{t}(\omega)=\sum I_{n}\left(f_{t}^{(n)}\right)$. From [H, prop. 4.5], we know that $X_{t}$ is adapted to $\mathcal{F}_{t}$ if and only if supp $f_{t}^{(n)} \subset[0, t]^{n}$. We define:

Definition 1 A generalized stochastic process $\Phi_{t}$ is said to be adapted if its kernels $F_{t}^{(n)}$ have the property $\operatorname{supp} F_{t}^{(n)} \subset[0, t]^{n}$ for all $t \in I$.

Remark Note that if the generalized stochastic process $\Phi_{t}$ is adapted, the $\left(L^{2}\right)$ stochastic process $e^{-\lambda N} \Phi_{t}$ will be adapted to $\mathcal{F}_{t}$ in the usual sense.

\section{The Itô integral for generalized stochastic processes}

In this section we define the Itô integral for adapted generalized stochastic processes. In order to construct such an integral, it is convenient to bring in the notion of strong independence for generalized random variables: $\Phi, \Psi \in \mathcal{G}^{*}$ are called strongly independent, if there exist two intervals or halflines $I_{\Phi}$ and $I_{\Psi}$ whose intersection has Lebesgue measure zero, and so that for all $m, n \in \mathbb{N}_{0}$, the kernels $\Phi^{(m)}, \Psi^{(n)}$ have their (essential) support in $\left(I_{\Phi}\right)^{m},\left(I_{\Psi}\right)^{n}$. We mention in passing that the notion of strong independence has also been discussed in [GHLØUZ]. From a probabilistic point of view independence and strong independence for usual random variables are not too different, as the next result shows. 
Proposition 2 Let $X, Y$ be two independent random variables in $\left(L^{2}\right)$ which are measurable w.r.t. $\sigma_{[a,+\infty)}:=\sigma\left\{B_{s}, a \leq s<+\infty\right\}, a \in \mathbb{R}$. Then $Y$ has a version $\tilde{Y}$ in $\left(L^{2}\right)$ so that $X, \tilde{Y}$ are strongly independent.

Proof Let $\tau_{a}^{*}$ be the linear continuous bijection from $\mathcal{S}(\mathbb{R})$ into itself, given by $\tau^{*} \xi(t):=\xi(-t+2 a), t \in \mathbb{R} . \tau_{a}$ denotes the adjoint of $\tau_{a}^{*}$ acting (weakly continuously) on $\mathcal{S}^{\prime}(\mathbb{R})$. Since $\tau_{a}^{*}$ is an $L^{2}(\mathbb{R})$-isometry, the white noise measure $\mu$ is invariant under $\tau_{a}$ (since $\tau_{a}$ is weakly continuous it is a measurable transformation on $\left(\mathcal{S}^{\prime}(\mathbb{R}), \mathcal{F}\right)$ : this follows immediately by calculating the characteristic function of $\mu_{\tau_{a}}$. Define $\widetilde{Y}:=Y \circ \tau_{a}$. Then $\widetilde{Y}$ has the same law as $Y$ : let $g$ be any real-valued bounded, measurable function on the real line, then

$$
\begin{aligned}
E(g(\tilde{Y})) & =\int g\left(Y \circ \tau_{a}(\omega)\right) d \mu(\omega) \\
& =\int g(Y(\omega)) d \mu(\omega) \\
& =E(g(Y)) .
\end{aligned}
$$

On the other hand, by construction the kernels of $X$ and $\tilde{Y}$ have the desired support property and hence $X$ and $\tilde{Y}$ are strongly independent.

Remark It is clear that by a similar argument one can deal with any finite (or even countably infinite) independent family of random variables which are measurable with respect to the values of Brownian motion over a finite timeinterval.

Lemma 3 The pointwise product of random variables has a well-defined extension to pairs $\Phi, \Psi$ of strongly independent elements in $\mathcal{G}^{*}$ so that $\Phi \cdot \Psi \in \mathcal{G}^{*}$. Moreover, the following formula holds

$$
\Phi \cdot \Psi=\Phi \diamond \Psi .
$$

Proof We only need to show that the above formula holds for strongly independent $\Phi$ and $\Psi$ which have only a finite number of non-vanishing kernels. The general case follows then by an approximation argument, and the fact that the Wick product is continuous on $\mathcal{G}^{*}$. Let $\Phi^{(n)}, \Psi^{(n)}$. respectively, denote the kernels of $\Phi, \Psi$. Using the well-known formula for the product of Wiener integrals (e.g. [HKPS], Cor. 2.13) we compute as follows:

$$
\begin{aligned}
\Phi \cdot \Psi & =\sum_{m=0}^{\infty} \sum_{n=0}^{\infty} \sum_{k=0}^{n \wedge m} k !\left(\begin{array}{l}
m \\
k
\end{array}\right)\left(\begin{array}{l}
n \\
k
\end{array}\right) I_{n+m-2 k}\left(\Phi^{(m)} \widehat{\otimes}_{k} \Psi^{(n)}\right) \\
& =\sum_{m=0}^{\infty} \sum_{n=0}^{\infty} I_{n+m}\left(\Phi^{(m)} \widehat{\otimes} \Psi^{(n)}\right) \\
& =\Phi \diamond \Psi .
\end{aligned}
$$


In the last computation $\Phi^{(m)} \widehat{\otimes}_{k} \Psi^{(n)}$ denotes the symmetrization of

$$
\int_{\mathbb{R}^{k}} \Phi^{(m)}(u, \cdot) \Psi^{(n)}(u, \cdot) d u
$$

which - due to the assumption of strong independence - is zero unless $k=0$. Moreover, the well-known formula for the chaos expansion of the Wick product used above can be found e.g. in [PT].

Observe that if $\Phi, \Psi \in \mathcal{G}^{*}$ are strongly independent, then

$$
e^{-\lambda N}(\Phi \cdot \Psi)=e^{-\lambda N} \Phi \cdot e^{-\lambda N} \Psi \text {. }
$$

We are now ready to construct the Itô integral on $[0, T]$ for adapted generalized stochastic processes: A generalized adapted process $\phi_{t}$ is called simple if it is of the form

$$
\phi_{t}=\sum_{i=0}^{M} \phi_{i} \cdot \mathbf{1}_{\left[t_{i}, t_{i+1}\right)}(t)
$$

where $\left\{t_{i} ; i=1, \ldots, M\right\}$ is a partition of $[0, T]$, and $\phi_{i}$ is $\mathcal{F}_{t_{i}}$ - adapted. Observe that if $F_{i}^{(n)}$ is the $n^{\prime}$ th kernel of $\phi_{i}$, then supp $F_{i}^{(n)} \subset\left[0, t_{i}\right]^{n}$. Hence, $\phi_{i}$ and $\left(B_{t_{i+1}}-B_{t_{i}}\right)$ are strongly independent. Therefore we can define the Itô integral of $\phi_{t}$ as

$$
\int_{0}^{T} \phi_{t} d B_{t}:=\sum_{i=0}^{M} \phi_{i} \cdot\left(B_{t_{i+1}}-B_{t_{i}}\right) .
$$

The following generalization of the Itô isometry holds: For $\lambda \geq 0$,

$$
\begin{aligned}
\left\|\int_{0}^{T} \phi_{t} d B_{t}\right\|_{-\lambda}^{2} & =\left\|e^{-\lambda N} \int_{0}^{T} \phi_{t} d B_{t}\right\|_{\left(L^{2}\right)}^{2} \\
& =\left\|\sum_{i}\left(e^{-\lambda N} \phi_{i}\right)\left(e^{-\lambda N}\left(B_{t_{i+1}}-B_{t_{i}}\right)\right)\right\|_{\left(L^{2}\right)}^{2} \\
& =\sum_{i, j} E\left[\left(e^{-\lambda N} \phi_{i}\right)\left(e^{-\lambda N} \phi_{j}\right)\left(e^{-\lambda}\left(B_{t_{i+1}}-B_{t_{i}}\right)\right) \times\right. \\
& \left.\times\left(e^{-\lambda}\left(B_{t_{j+1}}-B_{t_{j}}\right)\right)\right] \\
& =\epsilon^{-2 \lambda} \sum_{i} E\left[\left(e^{-\lambda N} \phi_{i}\right)^{2}\left(B_{t_{i+1}}-B_{t_{i}}\right)^{2}\right] \\
& =e^{-2 \lambda} \sum_{i} E\left[\left(e^{-\lambda N} \phi_{i}\right)^{2}\right]\left(t_{i+1}-t_{i}\right) \\
& =e^{-2 \lambda} \int_{0}^{T}\left\|\phi_{t}\right\|_{-\lambda}^{2} d t .
\end{aligned}
$$

Assume that $\Phi_{t}$ is an adapted generalized stochastic process which satisfies

$$
\int_{0}^{T}\left\|\Phi_{t}\right\|_{-\lambda}^{2} d t<\infty
$$


Such generalized processes will be called generalized Itô integrable processes. Note that $e^{-\lambda N} \Phi_{t} \in\left(L^{2}\right)$ is Itô integrable in the usual sense. Hence, there exists a sequence of simple stochastic processes $\left\{\phi_{t}^{k}\right\}_{k}$ in $\left(L^{2}\right)$ such that

$$
\int_{0}^{T}\left\|\phi_{t}^{k}-e^{-\lambda N} \Phi_{t}\right\|_{\left(L^{2}\right)}^{2} d t \rightarrow 0, \text { when } k \rightarrow 0
$$

I.e.,

$$
\int_{0}^{T}\left\|e^{\lambda N} \phi_{t}^{k}-\Phi_{t}\right\|_{-\lambda}^{2} d t \rightarrow 0, \text { when } k \rightarrow 0
$$

$e^{\lambda N} \phi_{t}^{k}$ is a generalized simple process. By the generalized Ito isometry, the sequence $\left\{\int_{0}^{T}\left(e^{\lambda N} \phi_{t}^{k}\right) d B_{t}\right\}_{k}$ is Cauchy in $\mathcal{G}_{-\lambda}$. We therefore define

$$
\int_{0}^{T} \Phi_{t} d B_{t}:=\lim _{k \rightarrow \infty} \int_{0}^{T} e^{\lambda N} \phi_{t}^{k} d B_{t}
$$

where the limit is taken in $\mathcal{G}_{-\lambda}$. Obviously, the generalized Itô isometry extends to generalized Itô integrable processes. Moreover, it is easy to see that the generalized Itô integral is linear: If $\Phi_{t}, \Psi_{t}$ are two generalized Itô integrable processes and $\alpha, \beta$ are two real numbers, then

$$
\int_{0}^{T} \alpha \Phi_{t}+\beta \Psi_{t} d B_{t}=\alpha \int_{0}^{T} \Phi_{t} d B_{t}+\beta \int_{0}^{T} \Psi_{t} d B_{t}
$$

Next we consider the relation between the generalized and the usual Itô integral.

Proposition 4 Let $\Phi_{t}$ be a generalized Itô integrable stochastic process with values in $\mathcal{G}_{-\lambda}, \lambda \geq 0$. Then

$$
\int_{0}^{T} \Phi_{t} d B_{t}=e^{\lambda(N-1)} \int_{0}^{T}\left(e^{-\lambda N} \Phi_{t}\right) d B_{t} .
$$

The integral on the right hand side is the usual Itô integral of the $L^{2}(\mu)$-valued process $\exp (-\lambda N) \Phi_{t}$. Moreover, if the kernels of $\Phi_{t}$ are given by the sequence $\left(F^{(n)}(t, \cdot), n \in N_{0}\right)$, then the generalized Ito integral has the following chaos decomposition:

$$
\int_{0}^{T} \Phi_{t} d B_{t}=\sum_{n=0}^{\infty} \frac{1}{n+1} I_{n}\left(\mathbf{1}_{[0, T)}^{\otimes n} \widehat{F^{(n)}}\right)
$$

where $\widehat{F^{(n)}}$ is the symmetrization of $F^{(n)}$ in all $n+1$ variables, and

$$
\mathcal{S}\left(\int_{0}^{T} \Phi_{t} d B_{t}\right)(\xi)=\int_{0}^{T} \mathcal{S} \Phi_{t}(\xi) \xi(t) d t .
$$


Proof First of all one shows equation (1) for simple processes by a straightforward computation using strong independence. Then this equality extends of course to generalized Itô integrable processes by their construction.

Next let $\left\{\phi_{t}^{k}\right\}_{k}$ be the sequence of simple processes in $\left(L^{2}\right)$ such that

$$
\int_{0}^{T}\left\|\phi_{t}^{k}-e^{-\lambda N} \Phi_{t}\right\|_{\left(L^{2}\right)}^{2} d t \rightarrow 0, \text { when } k \rightarrow \infty
$$

By definition,

$$
\left\|\int_{0}^{T} \phi_{t}^{k} d B_{t}-\int_{0}^{T} e^{-\lambda N} \Phi_{t} d B_{t}\right\|_{\left(L^{2}\right)}^{2} \rightarrow 0, \text { when } k \rightarrow \infty
$$

Hence,

$$
\begin{aligned}
& \left\|\int_{0}^{T} \phi_{t}^{k} d B_{t}-\int_{0}^{T} e^{-\lambda N} \Phi_{t} d B_{t}\right\|_{\left(L^{2}\right)}^{2} \\
& =\left\|e^{\lambda N} \int_{0}^{T} \phi_{t}^{k} d B_{t}-e^{\lambda N} \int_{0}^{T} e^{-\lambda N} \Phi_{t} d B_{t}\right\|_{-\lambda}^{2} \\
& =\left\|e^{\lambda} \int_{0}^{T} e^{\lambda N} \phi_{t}^{k} d B_{t}-e^{\lambda N} \int_{0}^{T} e^{-\lambda N} \Phi_{t} d B_{t}\right\|_{-\lambda}^{2}
\end{aligned}
$$

where we in the last equality have used formula (1). We see that

$$
e^{\lambda} \int_{0}^{T} e^{\lambda N} \phi_{t}^{k} d B_{t} \rightarrow e^{\lambda N} \int_{0}^{T} e^{-\lambda N} \Phi_{t} d B_{t}, \text { when } k \rightarrow \infty
$$

in $\mathcal{G}_{-\lambda}$. But by definition

$$
\int_{0}^{T} e^{\lambda N} \phi_{t}^{k} d B_{t} \rightarrow \int_{0}^{T} \Phi_{t} d B_{t}, \text { when } k \rightarrow \infty,
$$

The chaos decomposition for the generalized Itô integral follows now from the well-known formula for the chaos decomposition of the usual Itô integral. In the same way one finds the formula for its $S$-transform.

Remark The Itô integral defined above for generalized stochastic processes coincides with the Hitsuda-Skorohod integral (see e.g. [HKPS] for more information about the Hitsuda-Skorohod integral).

We end this section with an example: It is well-known that the Donsker $\delta$-function has chaos expansion for $x \in \mathbb{R}$ and $t>0$ given by

$$
\delta_{x}\left(B_{t}\right)=(2 t)^{-1 / 4} p_{t}(x)^{\frac{1}{2}} \sum_{n=0}^{\infty}\left(n ! t^{n}\right)^{-\frac{1}{2}} \xi_{n}\left(\frac{x}{\sqrt{2 t}}\right) I_{n}\left(1_{[0, t)}^{\otimes n}\right) .
$$


(e.g. [HKPS].) $p_{t}(x)$ is the heat kernel and $\xi_{n}$ is the $n$ 'th Hermite polynomial. By direct estimation, one finds (e.g. [PT]);

$$
\left\|\delta_{x}\left(B_{t}\right)\right\|_{-\lambda}^{2} \leq K_{\lambda} t^{-1}
$$

for all $\lambda>0 . K_{\lambda}$ is a positive number only depending on $\lambda$. Hence, $\delta_{x}\left(B_{t}\right)$ is a generalized process which is Itô integrable on $[s, T], s>0$. I.e.,

$$
\int_{s}^{T} \delta_{x}\left(B_{t}\right) d B_{t} \in \mathcal{G}_{-\lambda}, \text { for all } \lambda>0
$$

and

$$
\left\|\int_{s}^{T} \delta_{x}\left(B_{t}\right) d B_{t}\right\|_{-\lambda}^{2}=e^{-2 \lambda} \int_{s}^{T}\left\|\delta_{x}\left(B_{t}\right)\right\|_{-\lambda}^{2} d t
$$

\section{The martingale property for generalized pro- cesses}

In this section we consider the martingale property of generalized stochastic processes. To this end we first consider conditional expectations in $\mathcal{G}^{*}$.

Let $\mathcal{M}$ denote some subset of $\mathcal{S}(\mathbb{R})$, and let $P$ be the orthognal projection (in $L^{2}(\mathbb{R})$ ) of the $L^{2}(\mathbb{R})$-closure of the span of $\mathcal{M} . \mathcal{F}_{\mathcal{M}}$ denotes the sub- $\sigma$-algebra of $\mathcal{F}$ generated by $\mathcal{M}$, i.e. by the set of random variables $\{\langle\cdot, \xi\rangle ; \xi \in \mathcal{M}\}$. It is a basic exercise (left to the interested reader) to prove the following generalization of Proposition 4.7 in $[\mathrm{H}]$ :

Proposition 5 For $\phi \in L^{2}(\mu)$ with kernels $\phi^{(n)}$ the conditional expectation $E\left(\phi \mid \mathcal{F}_{\mathcal{M}}\right)$ is given by

$$
E\left(\phi \mid \mathcal{F}_{\mathcal{M}}\right)=\sum_{n=0}^{\infty} I_{n}\left(P^{\otimes n} \phi^{(n)}\right)
$$

i.e., $E\left(\cdot \mid \mathcal{F}_{\mathcal{M}}\right)=\Gamma(P)$, where $\Gamma$ denotes second quantization. In particular, conditional expectation w.r.t. $\mathcal{F}_{\mathcal{M}}$ maps the $n$-th chaos subspace of $L^{2}(\mu)$ into itself and it commutes with the chaos expansion.

Remark For a general sub- $\sigma$-algebra $\tilde{\mathcal{F}}$ of $\mathcal{F}$ the above statement that conditional expectation maps the $n$-th chaos into itself is false, as the following simple example shows (details are left to the interested reader): consider $B(1)$, i.e. Brownian motion at time 1 . Let $\widetilde{\mathcal{F}}$ be the sub- $\sigma$-algebra generated by $\operatorname{sign}(B(1))$, which obviously is not generated by random variables in the first chaos. Note furthermore that $\operatorname{sign}(B(1))$ and $|B(1)|$ are independent. Thus we have $E(B(1) \mid \widetilde{\mathcal{F}})=(2 / \pi)^{1 / 2} \operatorname{sign}(B(1))$, which clearly does not belong to the first chaos.

Let $\mathcal{F}_{\mathcal{M}}$ be a sub- $\sigma$-algebra generated by $\mathcal{M}$ as above. Then we can make the following definition. 
Definition 6 For every element $\Phi \in \mathcal{G}^{*}$, its conditional expectation with respect to $\mathcal{F}_{\mathcal{M}}$ is the element $E\left(\Phi \mid \mathcal{F}_{\mathcal{M}}\right)$ in $\mathcal{G}^{*}$ given by

$$
E\left(\Phi \mid \mathcal{F}_{\mathcal{M}}\right)=\sum_{n=0}^{\infty} I_{n}\left(P^{\otimes n} \Phi^{(n)}\right)
$$

where $\Phi^{(n)}, n \in \mathbb{N}_{0}$, are the kernels of $\Phi$.

We list some of the obvious properties of this generalized conditional expectation.

Lemma 7 For every $\lambda \in \mathbb{R}, E\left(\cdot \mid \mathcal{F}_{\mathcal{M}}\right)$ maps $\mathcal{G}_{\lambda}$ into itself. Furthermore, if $\Phi, \Psi \in \mathcal{G}^{*}$ and $a, b \in \mathbb{R}$, then the following properties hold:

(i) $E\left(a \Phi+b \Psi \mid \mathcal{F}_{\mathcal{M}}\right)=a E\left(\Phi \mid \mathcal{F}_{\mathcal{M}}\right)+b E\left(\Psi \mid \mathcal{F}_{\mathcal{M}}\right)$.

(ii) $E\left(E\left(\Phi \mid \mathcal{F}_{\mathcal{M}}\right)\right)=E(\Phi)$, where $E(\Phi):=\langle\langle\Phi, 1\rangle\rangle$.

(iii) $E\left(\Phi \mid \mathcal{F}_{\mathcal{M}}\right)=\Phi$ if $\Phi$ is $\mathcal{F}_{\mathcal{M}}$-measurable.

(iv) For all $\lambda \in \mathbb{R}, e^{\lambda N} E\left(\Phi \mid \mathcal{F}_{\mathcal{M}}\right)=E\left(e^{\lambda N} \Phi \mid \mathcal{F}_{\mathcal{M}}\right)$.

From now on we restrict our attention to the filtration $\left\{\mathcal{F}_{t}, t \geq 0\right\}$ of sub- $\sigma-$ algebras $\mathcal{F}_{t}$, generated by the standard version of Brownian motion $B_{t}$. (In order to phrase this as above, we choose for $t>0, \mathcal{M}$ to be the subset of functions in $\mathcal{S}(\mathbb{R})$ which have support in $[0, t]$, and the resulting $\sigma$-algebra coincides with $\mathcal{F}_{t}$.) In particular, we can now define the martingale property of generalized stochastic processes:

Definition 8 Let $M_{t}$ be a generalized process. It is called a martingale (w.r.t. $\left\{\mathcal{F}_{t}, t \geq 0\right\}$ ) if for all $t \geq s \geq 0$ the following holds:

$$
E\left(M_{t} \mid \mathcal{F}_{s}\right)=M_{s}
$$

(where the equality is in the sense of $\mathcal{G}^{*}$ ).

One part of the next equivalence is the generalization of Theorem 4.6 in [H] to $\mathcal{G}^{*}$-valued martingales.

Proposition 9 Let $M_{t}$ be a generalized stochastic process. Then the following are equaivalent:

(i) $M_{t}$ is a martingale.

(ii) For $\lambda$ large enough so that $e^{-\lambda N} M_{t} \in L^{2}(\mu), e^{-\lambda N} M_{t}$ is an $L^{2}(\mu)-$ martingale. 
(iii) For every $t \geq 0, M_{t}$ has a chaos expansion of the form

$$
M_{t}=\sum_{n=0}^{\infty} I_{n}\left(\mathbf{1}_{[0, t)}^{\otimes n} M^{(n)}\right)
$$

where $M^{(0)}$ is a real number, and for $n \in \mathbb{N}, M^{(n)}$ belongs to the symmetric subspace of $L_{\mathrm{loc}}^{2}\left(\mathbb{R}^{n}\right)$.

Proof That (i) and (ii) are equivalent is implied by the facts that $e^{-\lambda N}$ is a bijection from $\mathcal{G}_{\lambda}$ onto $L^{2}(\mu)$ (with inverse given by $e^{\lambda N}$ ), and that it commutes with the conditional expectation. The equivalence of (ii) and (iii) follows from Theorem 4.6 in $[\mathrm{H}]$, and again the fact that $e^{\lambda N}, \lambda \in \mathbb{R}$, commutes with the conditional expectation.

We list some results:

Corollary 10 Let $N_{t}$ be a generalized Itô integrable process. Then

$$
\int_{0}^{t} N_{s} d B_{s}
$$

is a martingale.

Proof By Proposition 4,

$$
e^{-\lambda N} \int_{0}^{t} N_{s} d B_{s}=e^{-\lambda} \int_{0}^{t}\left(e^{-\lambda N} N_{s}\right) d B_{s}
$$

which we know is a martingale in $L^{2}(\mu)$. Hence, the corollary follows from Proposition 9.

Corollary 11 Let $M_{t}$ be a martingale. Then for $t>s$

$$
\left\|M_{t}-M_{s}\right\|_{-\lambda}^{2}=\left\|M_{t}\right\|_{-\lambda}^{2}-\left\|M_{s}\right\|_{-\lambda}^{2} .
$$

Proof The corollary follows since $e^{-\lambda N} M_{t}$ is a martingale in $L^{2}(\mu)$ by Proposition 9 .

It is well-known that martingales in $\left(L^{2}\right)$ have orthogonal increments. For generalized martingales a similar results holds:

Corollary 12 Let $M_{t}$ be a $\mathcal{G}_{\lambda}$-martingale. If $\phi_{t} \in \mathcal{G}_{\lambda}$ is an $\mathcal{F}_{t}$-adapted process $(\lambda \geq 0)$, then for $t>s$

$$
\left\langle\left\langle M_{t}-M_{s}, \phi_{s}\right\rangle\right\rangle=0
$$

where $\langle\langle\cdot, \cdot\rangle\rangle$ is the dual pairing between $\mathcal{G}_{-\lambda}$ and $\mathcal{G}_{\lambda}$. 
Proof Since $\phi_{s}$ is $\mathcal{F}_{s}$-adapted, it has kernels with the property supp $\phi_{s}^{(n)} \mathrm{C}$ $[0, s]^{n}$. The kernels of $M_{t}-M_{s}$ are $\left(\mathbf{1}_{[0, t)}^{\otimes n}-\mathbf{1}_{[0, s)}^{\otimes n}\right) M^{(n)}$, which are zero on $[0, s]^{n}$. For generalized stochastic processes which are martingales, we have a martingale representation theorem:

Theorem 13 Let $M_{t}$ be a martingale with $M_{0}=0$ and chaos expansion

$$
M_{t}=\sum_{n=1}^{\infty} I_{n}\left(\mathbf{1}_{[0, t)}^{\otimes n} M^{(n)}\right)
$$

Then

$$
M_{t}=\int_{0}^{t} N_{s} d B_{s}
$$

where $N_{t}$ is an adapted generalized stochastic process with chaos expansion

$$
N_{t}=\sum_{n=0}^{\infty} I_{n}\left((n+1) \mathbf{1}_{[0, t)}^{\otimes n} M^{(n+1)}(t, \cdot)\right) .
$$

Moreover,

$$
\int_{0}^{t}\left\|N_{s}\right\|_{-\lambda}^{2} d s=e^{2 \lambda}\left\|M_{t}\right\|_{-\lambda}^{2} .
$$

Proof The proof goes by an application of the $\mathcal{S}$-transform on $M_{t}$ :

$$
\begin{aligned}
\mathcal{S} M_{t}(\xi) & =\sum_{n=1}^{\infty} \int_{[0, t) n} \xi^{\otimes n}\left(s_{1}, \ldots, s_{n}\right) M^{(n)}\left(s_{1}, \ldots, s_{n}\right) d s_{1} \cdots d s_{n} \\
& =\int_{0}^{t} \xi(s)\left(\sum_{n=1}^{\infty} n \int_{[0, s)^{n-1}} \xi^{\otimes n-1}\left(s_{1}, \ldots, s_{n-1}\right) \times\right. \\
& \left.M^{(n)}\left(s_{1}, \ldots, s_{n-1}, s\right) d s_{1} \cdots d s_{n-1}\right) d s \\
= & \int_{0}^{t} S N_{s}(\xi) \xi(s) d s .
\end{aligned}
$$

In the second equality we used the theorem of Fubini-Tonelli.

A corollary of this theorem is the result that the Wick product - unlike the ordinary product - preserves the martingale property:

Corollary 14 Let $\Phi_{t}$ and $\Psi_{t}$ be two martingales. Then $\Phi_{t} \diamond \Psi_{t}$ is a martingale.

Proof For the sake of convenience, assume that $\Phi_{0}=\Psi_{0}=0$. By the martingale representation theorem, we have

$$
\Phi_{t}=\int_{0}^{t} F_{s} d B_{s}, \quad \text { and } \Psi_{t}=\int_{0}^{t} G_{s} d B_{s}
$$


for two adapted generalized processes $F_{t}$ and $G_{t}$. We calculate,

$$
\begin{aligned}
\Phi_{t} \diamond \Psi_{t} & =\int_{0}^{t} F_{s} d B_{s} \diamond \int_{0}^{t} \Psi_{s} d B_{s} \\
& =\int_{0}^{t} F_{s} \diamond \int_{0}^{s} G_{u} d B_{u} d B_{s}+\int_{0}^{t} G_{s} \diamond \int_{0}^{s} F_{u} d B_{u} d B_{s} \\
& =\int_{0}^{t} F_{s} \diamond \Psi_{s} d B_{s}+\int_{0}^{t} G_{s} \diamond \Phi_{s} d B_{s} .
\end{aligned}
$$

In the second equality we used an integration by parts formula shown in [G] and $[\mathrm{BG}]$.

We turn our attention to integration with respect to martingales. If $M_{t}$ is a zero mean martingale in $\left(L^{2}\right)$ with the representation

$$
M_{t}=\int_{0}^{t} N_{s} d B_{s}
$$

we know from e.g. $[\mathrm{K}]$ that the integral with respect to $M_{t}$ of an adapted process $X_{t}$ with $E\left[\int_{0}^{t} X_{s}^{2} d\langle M\rangle_{s}\right]<\infty$ is given by

$$
\int_{0}^{t} X_{s} d M_{s}=\int_{0}^{t} X_{s} \cdot N_{s} d B_{s}
$$

Observe that $E\left[\int_{0}^{t} X_{s}^{2} d\langle M\rangle_{s}\right]=\int_{0}^{t}\left\|X_{s} \cdot N_{s}\right\|_{\left(L^{2}\right)}^{2} d s$. We can introduce integration with respect to a generalized martingale $M_{t}$ in the same manner, but now for a much more restricted class of integrands $X_{s}$. For a $\lambda>0$, let $M_{t}$ be a martingale in $\mathcal{G}_{-\lambda}$, which can be represented as the Itô integral of an adapted generalized process $N_{s}\left(N_{s} \in \mathcal{G}_{-\lambda}\right)$. Fix a $\nu>\frac{1}{2} \ln (2+\sqrt{2})$. Note that if $\Phi \in \mathcal{G}_{-\lambda}$ and $\phi \in \mathcal{G}_{\lambda+\nu}$, then

$$
\|\Phi \cdot \phi\|_{-(\lambda+\nu)} \leq C_{\nu}\|\Phi\|_{-\lambda}\|\phi\|_{\lambda+\nu} .
$$

(See e.g. [PT]). Hence, it is natural to define the integral with respect to $M_{t}$ for adapted processes $\phi_{s} \in \mathcal{G}_{\lambda+\nu}$ which fulfill

$$
\int_{0}^{t}\left\|\phi_{s} \cdot N_{s}\right\|_{-(\lambda+\nu)}^{2} d s<\infty
$$

We define for such processes

$$
\int_{0}^{t} \phi_{s} d M_{s}:=\int_{0}^{t} \phi_{s} \cdot N_{s} d B_{s}
$$

By the generalized Itô isometry we obtain

$$
\left\|\int_{0}^{t} \phi_{s} d M_{s}\right\|_{-(\lambda+\nu)}^{2}=e^{-2(\lambda+\nu)} \int_{0}^{t}\left\|\phi_{s} \cdot N_{s}\right\|_{-(\lambda+\nu)}^{2} d s
$$


It is easy to see that $\int_{0}^{t} \phi_{s} d M_{s}$ is a martingale.

We end this section with a discussion of sub- and supermartingales. We recall that an element $\Phi$ in $\mathcal{G}^{*}$ is called positive, denoted $\Phi \geq 0$, if it maps the positive cone of $\mu$-a.e. positive elements in $\mathcal{G}$ into $\mathbb{R}_{+}$. It is natural to make the following definition.

Definition 15 Let $\Phi_{t}$ be a generalized process. It is called a submartingale (a supermartingale, respectively) (w.r.t. $\left\{\mathcal{F}_{t}, t \geq 0\right\}$ ) if the following holds for all $t \geq s \geq 0$ :

$$
E\left(\Phi_{t} \mid \mathcal{F}_{s}\right)-\Phi_{s} \geq 0 \quad(\leq 0, \text { resp. }) .
$$

Proposition 16 Let $\Phi_{t}$ be a submartingale. Then for all $\lambda \geq 0, e^{-\lambda N} \Phi_{t}$ is a submartingale. In particular, if for some $\lambda \geq 0, \Phi_{t}$ is a $\mathcal{G}_{-\lambda}-$ valued submartingale, then $e^{-\lambda N} \Phi_{t}$ is an $L^{2}(\mu)$-submartingale. Analogous statements hold for supermartingales.

Proof The proposition follows from the (trivial) remark that the positivity preserving property of the Ornstein-Uhlenbeck semigroup $\left\{e^{-\lambda N}, \lambda \geq 0\right\}$ on $L^{2}(\mu)$ extends to $\mathcal{G}^{*}:$ if $\Phi \geq 0, \lambda \geq 0$, and $\varphi \geq 0(\mu$-a.e. $)$, then $\left\langle\left\langle e^{-\lambda N} \Phi, \varphi\right\rangle\right\rangle=$ $\left\langle\left\langle\Phi, e^{-\lambda N} \varphi\right\rangle\right\rangle \geq 0$, where we used that $e^{-\lambda N} \varphi \geq 0(\mu-$ a.s. $)$, e.g. [Si].

Remark In contrast to the situation of martingales, one does not have in general equivalence between the facts that a generalized process is a submartingale and that its regularization by the Ornstein-Uhlenbeck semigroup is an $L^{2}(\mu)$ submartingale. Here is a simple counterexample: Consider the process $\Phi_{t}$ in $\mathcal{G}^{*}$ (actually in $L^{2}(\mu)$ ) given by $\Phi_{t}=e^{\lambda N} B_{t}^{2}$, where $\lambda>0$ and $B_{t}$ - as before - is Brownian motion at time $t$. Obviously $e^{-\lambda N} \Phi_{t}$ is an $L^{2}(\mu)$-submartingale. On the other hand, we have for $t \geq s \geq 0$ :

$$
E\left(\Phi_{t} \mid \mathcal{F}_{s}\right)-\Phi_{s}=t-e^{2 \lambda} s,
$$

which is strictly negative for $s \in\left(e^{-2 \lambda} t, t\right]$

\section{Generalized stochastic processes and contin- uous semimartingales}

In this section we discuss the semimartingale property for generalized stochastic processes. We will define this property, and look at some examples. First we need to generalize the notion of pathwise bounded variation and continuity:

Definition 17 Let $A_{t}$ be a generalized stochastic process. We say that $t \mapsto A_{t}$ is of bounded variation on every finite interval $[0, t], t>0$, if $t \mapsto e^{-\lambda N} A_{t}$ is of bounded variation on every finite interval $[0, t]$.

Pathwise continuity is defined in the same manner: 
Definition 18 Let $\Phi_{t}$ be a generalized stochastic process. $\Phi_{t}$ is said to be continuous if $e^{-\lambda N} \Phi_{t}$ is pathwise continuous. (i.e. $t \mapsto e^{-\lambda N} \Phi_{t}(\omega)$ is continuous a.s.).

We prove that continuous martingales cannot be of bounded variation:

Proposition 19 Let $M_{t}$ be a continuous martingale with $M_{0}=0$. Assume $M_{t}$ is of bounded variation on every finite interval $[0, t]$. Then $M_{t}=0$.

Proof By definition, $e^{-\lambda N} M_{t}$ is a continuous martingale in $\left(L^{2}\right)$ which is of bounded variation on every finite interval. From Th. 2.6.2 in $[\mathrm{K}], e^{-\lambda N} M_{t}=0$ a.s. Hence, $M_{t}=e^{\lambda N}\left(e^{-\lambda N} M_{t}\right)=0$.

This proposition enables us to extend the notion of continuous semimartingale to generalized stochastic processes: (This is an extension of the definition 4.1.1 found in $[\mathrm{K}]$. See also $[\mathrm{RY}]$.)

Definition 20 A continuous generalized stochastic process $\Phi_{t}$ is suid to be a continuous. semimartingale if it is of the form

$$
\Phi_{t}=\Phi_{0}+M_{t}+A_{t}
$$

where $M_{t}$ is a continuous martingale with $M_{0}=0 . A_{t}$ is a continuous generalized stochastic process of bounded variation on every finite interval, and $A_{0}=0$.

In order to give some interesting examples of semimartingales, we need some results about the Bochner integral of a generalized process:

Proposition 21 Let $\Phi_{t}$ be a generalized stochastic process which satisfies the following on $[0, T]$ :

1. $t \mapsto\left\langle\left\langle\Phi_{t}, \phi\right\rangle\right\rangle$ is measurable for every $\phi \in \mathcal{G}$.

2. $\int_{0}^{T}\left\|\Phi_{t}\right\|_{-\lambda} d t<\infty$.

Then $e^{-\lambda N} \Phi_{t}$ is Bochner integrable in $\left(L^{2}\right)$ and

$$
e^{-\lambda N} \int_{0}^{T} \Phi_{t} d t=\int_{0}^{T} e^{-\lambda N} \Phi_{t} d t
$$

Moreover, $e^{-\lambda N} \Phi_{t}(\omega)$ is integrable on $[0, T]$ a.s. (pathwise integrable), and the Bochner integal of $e^{-\lambda N} \Phi_{t}$ coincides with the pathwise integral a.s.

Proof Assumption 1 and 2 imply that $\Phi_{t}$ is Bochner integrable in $\mathcal{G}_{-\lambda}$. Hence, $e^{-\lambda N} \Phi_{t}$ is Bochner integrable in $\left(L^{2}\right)$. Let $\Phi_{t}$ have kernels $\Phi_{t}^{(n)}(\cdot)$. We calculate:

$$
e^{-\lambda N} \int_{0}^{T} \Phi_{t} d t=e^{-\lambda N} \sum_{n=0}^{\infty} I_{n}\left(\int_{0}^{T} \Phi_{t}^{(n)} d t\right)
$$




$$
\begin{aligned}
& =\sum_{n=0}^{\infty} I_{n}\left(\int_{0}^{T} e^{-\lambda n} \Phi_{t}^{(n)} d t\right) \\
& =\int_{0}^{T} e^{-\lambda N} \Phi_{t} d t .
\end{aligned}
$$

We show that $e^{-\lambda N} \Phi_{t}(\omega) \in L^{1}([0, T], d t)$ a.s. Using Tonelli's theorem and Cauchy-Schwarz,

$$
\begin{aligned}
E\left[\int_{0}^{T}\left|e^{-\lambda N} \Phi_{t}\right| d t\right] & =\int_{0}^{T} E\left[\left|e^{-\lambda N} \Phi_{t}\right|\right] d t \\
& \leq \int_{0}^{T}\left\|e^{-\lambda N} \Phi_{t}\right\|_{\left(L^{2}\right)} d t \\
& =\int_{0}^{T}\left\|\Phi_{t}\right\|_{-\lambda} d t .
\end{aligned}
$$

Hence, by Fubini's theorem $e^{-\lambda N} \Phi_{t}(\omega) \in L^{1}([0, T], d t)$, a.s. The pathwise integral of $e^{-\lambda N} \Phi_{t}$ is in $\left(L^{2}\right)$, and coincides with the Bochner integral of $e^{-\lambda N} \Phi_{t}$. If $\Phi_{s}$ is a generalized stochastic process satisfying conditions 1 and 2 on $[0, t]$ for every $t>0$ in the above propostion, we see that $\int_{0}^{t} \Phi_{s} d s$ is of bounded variation on every finite interval $[0, t]$.

For $F \in \mathcal{S}^{\prime}(\mathbb{R})$, we want to show that $F \circ B_{t}$ is a semimartingale: We argue that $F \circ B_{t}$ is a generalized process: first, $F \circ B_{t} \in(\mathcal{S})^{*}$ for all $t>0$ (e.g. [HKPS], or as a consequence of Watanabe's theorem it is an element in $\mathcal{D}^{*} \subset(\mathcal{S})^{*}$.) Also, from equation (3.19) in [HKPS], we have

$$
F \circ B_{t}=\sum_{n=0}^{\infty} \frac{1}{n !}\left\langle F, \eta_{n, t}\right\rangle I_{n}\left(\mathbf{1}_{[0, t)}^{\otimes n}\right)
$$

where

$$
\eta_{n, t}(x)=g_{t}(x)\left(\frac{\pi^{\frac{1}{2}} n !}{t^{n}}\right)^{\frac{1}{2}} \xi_{n}\left(\frac{x}{\sqrt{2 t}}\right) .
$$

Here $\xi_{n}$ is the $n^{\prime}$ th Hermite function and

$$
g_{t}(x)=(2 \pi t)^{-\frac{1}{2}} e^{-\frac{x^{2}}{4 t}} .
$$

Note that both $g_{t}$ and $\xi_{n}$ are functions in the Schwartz space. Standard estimates give

$$
\begin{aligned}
\left|\left\langle F, \eta_{n, t}\right\rangle\right| & \leq K_{t, p}\left|\xi_{n}\right|_{2, p} \sqrt{2 t}\left(\frac{\pi^{\frac{1}{2}} n !}{t^{n}}\right)^{\frac{1}{2}} \\
& \leq K_{t, p}(2 n+1)^{p} \sqrt{2 t}\left(\frac{\pi^{\frac{1}{2}} n !}{t^{n}}\right)^{\frac{1}{2}}
\end{aligned}
$$


In the second inequality we used that $\xi_{n}$ is the eigenfunction of the harmonic oscillator $H=-\Delta+x^{2}+1$ with eigenvalue $2 n+1 . K_{t, p}$ is a positive number dependent on $K, t$, and $p$. 'We estimate for $\lambda>0$

$$
\begin{aligned}
\left\|F \circ B_{t}\right\|_{-\lambda}^{2} & =\sum_{n=0}^{\infty} n ! e^{-2 \lambda n}\left\langle F, \eta_{n, t}\right\rangle^{2}\left(\frac{1}{n !}\right)^{2} t^{n} \\
& =\sum_{n=0}^{\infty} \frac{t^{n}}{n !} e^{-2 \lambda n}\left\langle F, \eta_{n, p}\right\rangle^{2} \\
& \leq 2 t \pi^{\frac{1}{2}} K_{t, p}^{2} \sum_{n=0}^{\infty} \frac{(2 n+1)^{2 p}}{e^{2 \lambda n}} \\
& <\infty
\end{aligned}
$$

Hence, we have shown that $F \circ B_{t}$ is a generalized stochastic process. Fix $\epsilon>0$, and introduce the $\sigma$-algebra $\mathcal{F}_{t}^{\epsilon}=\sigma\left\{B_{s}: \epsilon \leq s \leq t\right\}$. For $t \geq \epsilon$, we have from the Itô Formula (Theorem 8.8 in [HKPS]),

$$
F \circ B_{t}-F \circ B_{\epsilon}=\int_{\epsilon}^{t} F^{\prime} \circ B_{s} d B_{s}+\frac{1}{2} \int_{\epsilon}^{t} F^{\prime \prime} \circ B_{s} d s,
$$

where $F^{\prime}$ and $F^{\prime \prime}$ are distributional derivatives. By a slight modification of the definitions of martingales and semimartingales introduced in this paper, we see that $F \circ B_{t}$ is a semimartingale with respect to $\mathcal{F}_{t}^{\epsilon}$.

\section{References}

[BG] F. E. Benth and H. Gjessing (1994): A Non Linear Parabolic Equation with Noise. A Reduction Method. Preprint, University of Mannheim, Germany.

[G] H. Gjessing (1994): Wick Calculus with Applications to Anticipating Stochastic Differential Equations. Preprint, University of Bergen, Norway.

[GHLøUZ] H. Gjessing, H. Holden, T. Lindstrøm, B. Øksendal, J Ubøe and T. -S. Zhang (1993): The Wick Product. In. H. Niemi et al. (eds.): Frontiers in Pure and Applied Probability, TVP Publishers, Moscow, pp. 29-67.

[H] T. Hida (1980): Brownian Motion. Springer Verlag.

[HKPS] T. Hida, H. -H. Kuo, J. Potthoff and L. Streit (1993): White Noise An Infinite Dimensional Calculus. Kluwer Academic Publishers.

[K] G. Kallianpur.(1980): Stochastic Filtering Theory. Springer Verlag.

[KK] G. Kallianpur and H.-H. Kuo (1984): Regularity Properties of Donsker's Delta Function. Appl. Math. Optim. 12, pp. 89-95. 
[PT] J. Potthoff and M. Timpel (1993): On a Dual Pair of Spaces of Smooth and Generalized Random Variables. Preprint, University of Mannheim, Germany, to appear in Potential Analysis.

[RY] D. Revuz and M. Yor (1994): Continuous Martingales and Brownian Motion. Second Edition. Springer Verlag.

[Si] B. Simon (1974): The $P(\phi)_{2}$ Euclidean (Quantum) Field Theory, Princeton University Press. 